\title{
Comparison of two different approaches for the analysis of data from a prospective cohort study: an application to work related risk factors for low back pain
}

\author{
W E Hoogendoorn, P M Bongers, H C W de Vet, J W R Twisk, W van Mechelen, \\ L M Bouter
}

See end of article for authors' affiliations

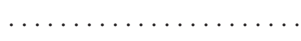

Correspondence to: Dr P M Bongers, TNO Work and Employment, PO Box 718, 2130 AS Hoofddorp, Netherlands: p.bongers@arbeid.tno.nl

Accepted 13 February 2002

\begin{abstract}
Aims: To compare the results of a traditional approach using standard regression for the analysis of data from a prospective cohort study with the results of generalised estimating equations (GEE) analysis.

Methods: The research was part of a three year prospective cohort study on work related risk factors for low back pain. The study population consisted of a cohort of 1192 workers with no low back pain at baseline. Information on work related physical and psychosocial factors and the occurrence of low back pain was obtained by means of questionnaires at baseline and at the three annual follow up measurements. In a traditional standard logistic regression model, physical and psychosocial risk factors at baseline were related to the cumulative incidence of low back pain during the three year follow up period. In a GEE logistic model, repeated measurements of the physical and psychosocial risk factors were related to low back pain reported at one measurement point later.

Results: The traditional standard regression model showed a significant effect of flexion and/or rotation of the upper part of the body (OR $=1.8 ; 95 \% \mathrm{Cl}: 1.2$ to 3.0$)$, but not of moving heavy loads $(O R=1.4 ; 95 \% \mathrm{Cl}: 0.7$ to 3.1$)$. The GEE model showed a significant effect of both flexion and/or rotation of the upper part of the body $(\mathrm{OR}=2.2 ; 95 \% \mathrm{Cl}: 1.5$ to 3.3$)$ and moving heavy loads $(O R=1.5 ; 95 \% \mathrm{Cl}: 1.0$ to 2.4$)$. No significant associations with low back pain were found for the psychosocial work characteristics with either method, but the GEE model showed weaker odds ratios for these variables than the traditional standard regression model.

Conclusions: Results show that there are differences between the two analytical approaches in both the magnitude and the precision of the observed odds ratios.
\end{abstract}

f $\mathrm{n}$ occupational health research on work related risk factors for musculoskeletal symptoms, there is an increasing awareness that prospective cohort studies are necessary to obtain more insight into the temporal relation between work related risk factors and health outcomes. ${ }^{1-4}$ Traditionally, these studies have mainly focused on examination of the relation between exposure measured at baseline and occurrence of the health outcome of interest during a specified follow up period. However, changes at the workplace may invalidate baseline data, and repeated measurements of exposure should therefore be considered. A recent review of studies on physical risk factors for back pain showed that hardly any cohort studies incorporated repeated measurements of exposure, although there were many studies with an extremely long follow up period during which the exposure could have changed considerably. ${ }^{5}$

Repeated measurements can be made of both exposures and outcomes. The generalised estimating equation (GEE) method developed by Liang and Zeger is one of the methods that can be used for the analysis of repeated measurements data. ${ }^{67}$ The GEE method extends standard regression analysis, taking into account the correlation between repeated measurements. The marginal distribution of the outcome is modelled, which means that GEE models describe the average occurrence of the outcome for the group as a whole over time. Data on a person at a certain time during follow up are included, whether or not data on that person are missing at other times, under the assumption that the pattern of missing data is random. The GEE method can be applied to a wide range of familiar models, including linear and logistic regression, which means that the method is suitable for the analysis of both continuous and dichotomous outcome variables. In addition, both time dependent and time independent covariates can be included. ${ }^{6-9}$ Gender is a typical example of a time independent variable. All variables that are subject to change are, in principle, time dependent.

The Study on Musculoskeletal disorders, Absenteeism, Stress, and Health (SMASH) was a three year prospective cohort study among a working population, which was initiated to identify risk factors for musculoskeletal disorders. In this study, repeated measurements were made of both exposures and outcomes. In previous reports on the study, a traditional approach was used for the data analysis, implying that work related risk factors at baseline were related to the cumulative incidence of low back pain reported during follow up in a standard regression. ${ }^{10}{ }^{11}$ The objective of the study described in this paper was to compare the results of this traditional approach with the results of a GEE analysis that includes the repeated measurements of exposure and outcome, to find out whether the latter approach produces different parameter and standard error estimates. For this purpose, both analytical approaches were applied to examine the relation between selected work related physical and psychosocial risk factors and the occurrence of low back pain.

Abbreviations: GEE, generalised estimating equations; JCQ, Job Content Questionnaire; SMASH, Study on Musculoskeletal disorders, Absenteeism, Stress, and Health 


\section{Main messages}

- The results of a traditional approach using standard regression analysis and generalised estimating equations differ in both magnitude and precision of the calculated associations.

- In the design and analysis of prospective cohort studies on work related risk factors for musculoskeletal symptoms, incorporation of repeated measurements of exposure should be considered.

- Longitudinal studies involving repeated measurements with relatively short time intervals should be performed to obtain more insight into (the time lag of) the effect of psychosocial work characteristics.

\section{Policy implications}

- Reduction of trunk flexion and trunk rotation at work can contribute to the prevention of low back pain.

- Reduction of moving heavy loads at work can contribute to the prevention of low back pain.

- The role of improvement of the psychosocial work environment by reducing quantitative demands and conflicting demands, and increasing supervisor and coworker support in the prevention of low back pain is less clear.

\section{PARTICIPANTS AND METHODS}

\section{Study population}

For the SMASH study, workers were recruited from 34 companies located throughout the Netherlands. The participating companies were asked to select workers who had been employed in their current job for at least one year and who were working for 24 hours per week or more. Workers in blue collar jobs, as well as workers in white collar jobs and caring professions were included in the study.

At baseline, $1789(87 \%)$ of the 2064 workers who were invited to participate, completed the questionnaire. Of these, 1738 were eligible for participation in the part of the study focusing on risk factors for low back pain..$^{10}$ For the analyses described in this paper, a cohort of 1192 workers with no low back pain at baseline was identified, consisting of workers who reported at baseline that they had not had regular or prolonged low back pain in the previous 12 months. ${ }^{111}$

\section{Baseline survey}

Between March 1994 and March 1995 the participants completed a questionnaire and underwent a physical exam- ination. The questionnaire included questions on the individual factors of age, gender, smoking habits, exercise behaviour, ${ }^{12}$ and coping skills. ${ }^{13}$ Assessment of the body mass index was based on measurements of weight and height taken by a physiotherapist during the physical examination.

Psychosocial work characteristics were assessed by means of a Dutch version of Karasek's Job Content Questionnaire (JCQ), ${ }^{14}$ which includes dimensions on quantitative job demands, decision authority, skill discretion, supervisor support, and coworker support. Conflicting demands were assessed on the basis of one single item from the JCQ. ${ }^{14}$

Flexion and/or rotation of the upper part of the body and moving heavy loads ( $>25 \mathrm{~kg}$ ) at work were assessed by means of the Loquest questionnaire. ${ }^{15}$ Driving a vehicle at work was also assessed with this questionnaire, and aspects of physical load during leisure time were assessed on the basis of questions comparable to those used to assess the physical load at work. ${ }^{15}$

Assessment of the occurrence of low back pain was based on an adaptation of the Nordic Questionnaire. ${ }^{16}$ Workers had to answer the question "Have you had trouble (aches, pain, discomfort) in the low back in the previous 12 months?" with one of the following four response options: no, never; yes, sometimes; yes, regularly; yes, prolonged. A case of low back pain was defined if a worker reported regular or prolonged low back pain in the previous 12 months.

\section{Follow up}

After the baseline survey there was a follow up period of three years, and each year the workers received a self administered questionnaire by post. In these questionnaires, the same questions as in the baseline questionnaire were asked to assess exercise behaviour, psychosocial work characteristics, physical load at work and during leisure time, and the occurrence of low back pain in the previous 12 months.

\section{Statistical analysis}

Two analytical methods were used: standard regression and GEE. Both methods were carried out with Proc Genmod in the statistical package SAS (version 6.12), ${ }^{17}$ and as the outcome variable low back pain is dichotomous, the link function in Proc Genmod was always specified as logistic. Figure 1 illustrates the models that were analysed with the two methods. The regression equations for these models are given in the appendix.

In the standard regression model, the baseline measurements of work related physical risk factors (flexion and/or rotation of the upper part of the body and moving heavy

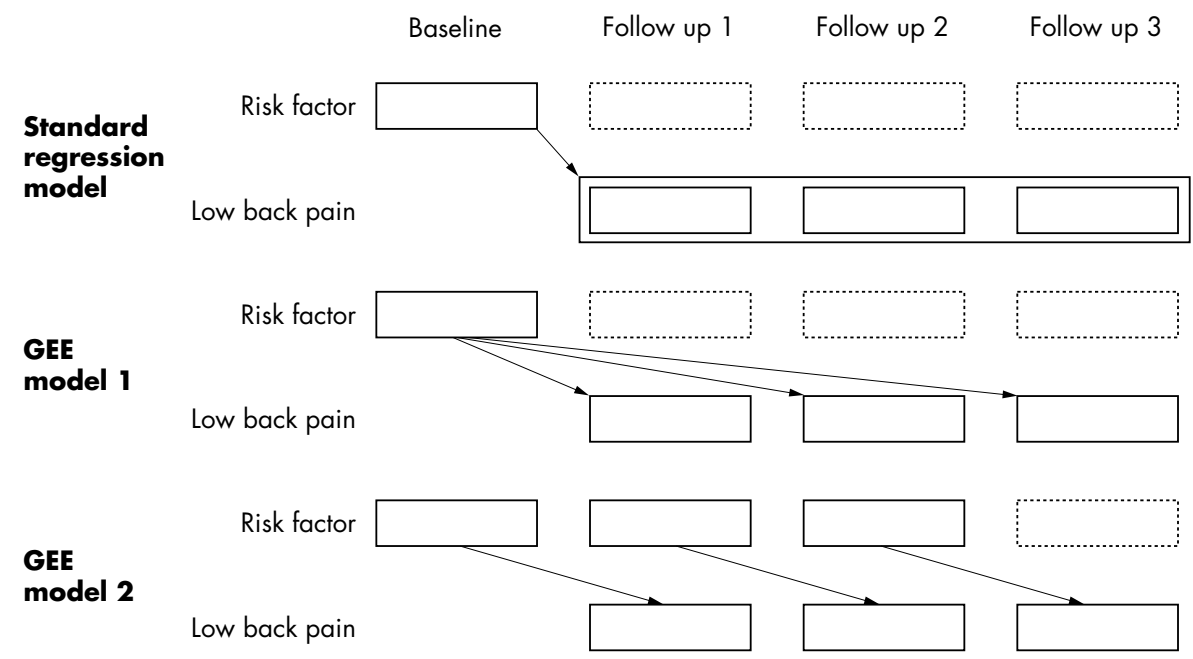

Figure 1 Illustration of the different models that were analysed with standard regression and generalised estimating equations (GEE). 
Table 1 Low back pain reported at the annual follow up measurements in the cohort of workers with no low back pain in the previous 12 months at baseline $(n=1192)$

\begin{tabular}{lll}
\hline Measurement & Low back pain (\%) & Missing data (n) \\
\hline Follow up 1 & 12.3 & 131 \\
Follow up 2 & 14.3 & 216 \\
Follow up 3 & 13.7 & 218 \\
Cumulative & 26.6 & 331 \\
\hline
\end{tabular}

loads) and work related psychosocial risk factors (quantitative job demands, conflicting demands, supervisor support, and coworker support) were related to the cumulative incidence of low back pain reported during the three year follow up period. In this analysis, a case of low back pain was defined if a worker reported, in at least one of the annual self administered follow up questionnaires, regular or prolonged low back pain in the previous 12 months.

Using GEE, ${ }^{67}$ two different models were analysed. In GEE model 1, all risk factors and covariates were assumed to be time independent, which means that only data from the baseline measurement were used, as in the standard regression analysis. These baseline measurements were related to low back pain reported at the three different follow up points. In GEE model 2, all risk factors were time dependent. Most covariates were also time dependent, except for age and gender and the covariates that were only measured at baseline (smoking habits, coping skills, and body mass index). GEE model 2 was also a time lag model, implying that the repeated measurements of the risk factors studied were related to low back pain reported at one measurement point later. ${ }^{79}$ Given the fact that in the present study, data on the occurrence of low back pain collected at each measurement point concerned the previous 12 months, the use of a time lag model was necessary to take into account the temporal sequence of cause and effect. GEE model 2 was considered a priori to be the most appropriate model for both the physical and the psychosocial risk factors studied, because it takes into account the time varying nature of both the outcome and the exposure. GEE model 1 was also analysed to be able to obtain insight into the specific effect of taking into account the time varying nature of the exposure by comparison of GEE models 1 and 2 .

The analyses of both GEE models were performed using an exchangeable working correlation structure, implying that all correlations between repeated observations of the outcome variable from each subject were assumed to be equal, irrespective of the time period between the measurements. ${ }^{679}$ This structure was chosen, because it is the most neutral option, and also because the relatively short follow up period of this study and the identical duration of the intervals between the repeated measurements does not warrant the use of a more specific correlation structure. ${ }^{79}$

In univariate analyses, performed with both analytical methods, crude odds ratios with corresponding 95\% confidence intervals were calculated for the risk factors studied. Adjusted odds ratios were determined in a full model, including the work related physical and psychosocial risk factors and the individual factors, other psychosocial work characteristics, other work related physical factors, and physical factors during leisure time that were considered as covariates. Both univariate and multivariable GEE models always included a linear time effect (from 0 to 2 ).

\section{RESULTS}

Table 1 shows the data on reported low back pain for each follow up measurement separately and combined. From the cohort of 1192 workers with no low back pain in the previous 12 months at baseline, data on the occurrence of low back pain were available for 861 workers (72\%) for all three annual follow up measurements. These data were used for the analyses with the standard regression method. The cumulative incidence of low back pain in this group during the three year follow up period was $26.6 \%$.

From the cohort of 1192 workers, data on the occurrence of low back pain were available for 1116 workers (94\%) for at least one of the follow up measurements. These data were used for the analyses with the GEE method. At each follow up measurement, approximately 13\% of workers reported that they had had regular or prolonged low back pain in the previous 12 months.

Table 2 gives a detailed overview of the number of reports of low back pain among workers, with data on the occurrence of

Table 2 Overview of the number of follow up measurements attended and the number of reports of low back pain among workers with data on the occurrence of low back pain for at least one of the follow up measurements $(n=1116)$

\begin{tabular}{llrrr}
\hline \multirow{2}{*}{$\begin{array}{l}\text { Number of follow up } \\
\text { measurements attended }\end{array}$} & \multicolumn{4}{l}{ Number of reports of low back pain (\%) } \\
\cline { 2 - 5 } & 0 & 1 & 2 & 3 \\
\hline 3 & $632(73.4)$ & $138(16.0)$ & $69(8.0)$ & $22(2.6)$ \\
2 & $132(76.3)$ & $32(18.5)$ & $9(5.2)$ & 861 \\
1 & $71(86.6)$ & $11(13.4)$ & & 82 \\
\hline
\end{tabular}

Table 3 Overview of changes in reported exposure between subsequent follow up measurements

\begin{tabular}{|c|c|c|c|c|c|c|}
\hline \multirow[b]{2}{*}{ Risk factor } & \multicolumn{3}{|c|}{$\begin{array}{l}\text { Change in exposure between baseline and } \\
\text { follow up } 1(\%)^{*}\end{array}$} & \multicolumn{3}{|c|}{$\begin{array}{l}\text { Change in exposure between follow up } 1 \\
\text { and follow up } 2(\%) \dagger\end{array}$} \\
\hline & 0 levels & 1 level & 2 levels & 0 levels & 1 level & 2 levels \\
\hline Flexion and/or rotation of the upper part of the body & $658(61.9)$ & $350(32.9)$ & $55(5.2)$ & $678(71.8)$ & $243(25.7)$ & $23(2.4)$ \\
\hline Moving heavy loads $(>25 \mathrm{~kg})$ & $876(82.3)$ & $171(16.1)$ & $18(1.7)$ & $812(85.3)$ & $118(12.4)$ & $22(2.3)$ \\
\hline Quantitative job demands & $682(64.2)$ & $372(35.0)$ & $8(0.8)$ & $628(66.2)$ & $312(32.9)$ & $9(0.9)$ \\
\hline Conflicting demands & $672(63.8)$ & $313(29.7)$ & $68(6.5)$ & $646(68.1)$ & $267(28.2)$ & $35(3.7)$ \\
\hline Supervisor support & $647(61.4)$ & $376(35.7)$ & $31(2.9)$ & $613(65.2)$ & $298(31.7)$ & $29(3.1)$ \\
\hline Coworker support & $746(70.6)$ & $295(27.9)$ & $16(1.5)$ & $662(69.5)$ & $271(28.5)$ & $19(2.0)$ \\
\hline
\end{tabular}

*This analysis is based on those workers that provided data on exposure to the specific risk factor, at both baseline and at follow up 1. †This analysis is based on those workers that provided data on exposure to the specific risk factor at both follow up 1 and follow up 2. 


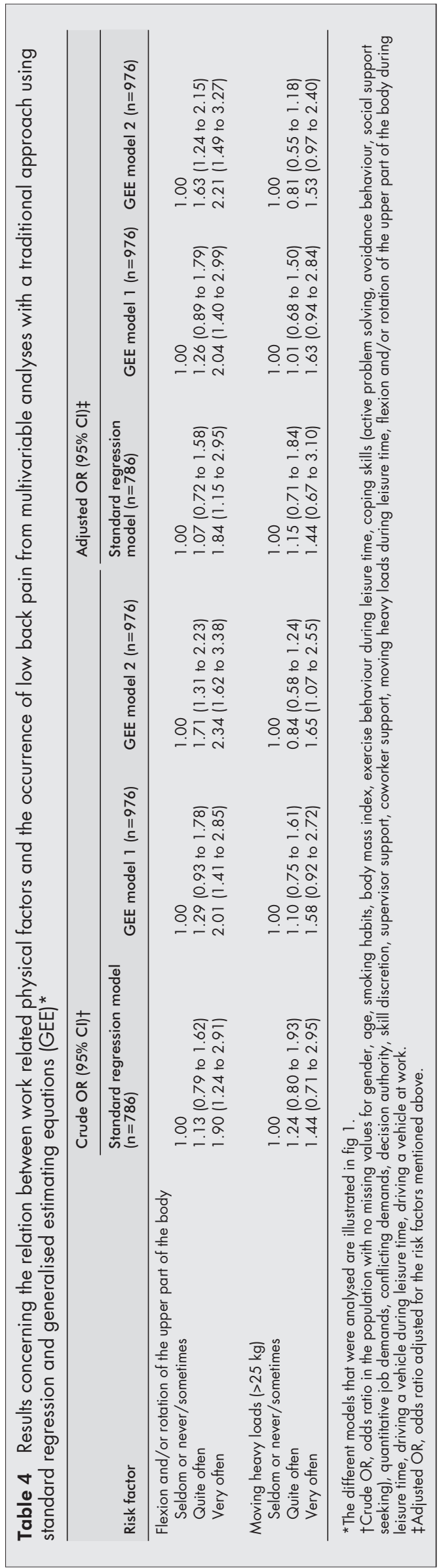

low back pain for at least one of the follow up measurements. Only $2.6 \%$ of the workers with data for all follow up measurements reported regular or prolonged low back pain in the previous 12 months at all three follow up measurements.

The inclusion of repeated measurements of exposure is only relevant when there is at least some change in the exposure over time. Table 3 gives an overview of changes in the reported exposure to the risk factors under study between subsequent measurements. In general, for no more than $5 \%$ of the workers exposure to a specific risk factor changed more than one level between two subsequent measurements.

Table 4 shows the results for the work related physical risk factors studied. A comparison of the crude results from GEE model 2 with the crude results from the traditional approach using standard regression shows that for the medium and the highest level of flexion and/or rotation of the upper part of the body the odds ratios in GEE model 2 were higher. In GEE model 2, a dose-response relationship was also observed. The odds ratios for flexion and/or rotation of the upper part of the body in GEE model 1 were lower than the odds ratios in GEE model 2. For the highest level of moving heavy loads the odds ratio was somewhat higher in GEE model 2, while for the medium level of moving heavy loads, the odds ratio in GEE model 2 was slightly lower. The odds ratios for moving heavy loads in GEE model 1 were comparable to those in GEE model 2. A comparison between the adjusted odds ratios resulting from the different analyses shows a similar pattern for both flexion and/or rotation of the upper part of the body and moving heavy loads. In general, the $95 \%$ confidence intervals were found to be relatively smaller in the GEE analyses than in the standard regression analyses. With the latter approach, a statistically significant relationship with low back pain was found for flexion and/or rotation of the upper part of the body, but not for moving heavy loads, while with GEE a (borderline) significant relationship with low back pain was found for both flexion and/or rotation of the upper part of the body and moving heavy loads.

Table 5 shows the results for the work related psychosocial risk factors studied. A comparison of the crude results from GEE model 2 with the crude results from the standard regression model shows that for both medium and high quantitative job demands the odds ratios in GEE model 2 were lower. The odds ratio for high quantitative demands was slightly higher in GEE model 1 than in GEE model 2. For high conflicting demands, the odds ratio in GEE model 2 was also lower than in the standard regression model, while for the medium level of conflicting demands the odds ratio in GEE model 2 was slightly higher. The odds ratios for conflicting demands in GEE model 1 were comparable to those in GEE model 2. For medium and low supervisor and coworker support, lower odds ratios were found in GEE model 2 than in the standard regression model. For supervisor support the odds ratios in GEE model 1 were comparable to the odds ratios in GEE model 2. For coworker support the odds ratios in GEE model 1 were slightly higher than the odds ratios in GEE model 2. A comparison between the adjusted odds ratios resulting from the different models shows a similar pattern for the work related psychosocial risk factors. Again, the 95\% confidence intervals were relative smaller in the GEE analyses. After adjustment for potential confounders, no statistically significant relationship with reported low back pain was found for any of the work related psychosocial risk factors studied.

\section{DISCUSSION}

\section{Summary and interpretation of findings}

In this article, two different analytical approaches were applied to longitudinal data from a prospective cohort study on work related risk factors for musculoskeletal symptoms. For the work related physical factors studied, higher odds 


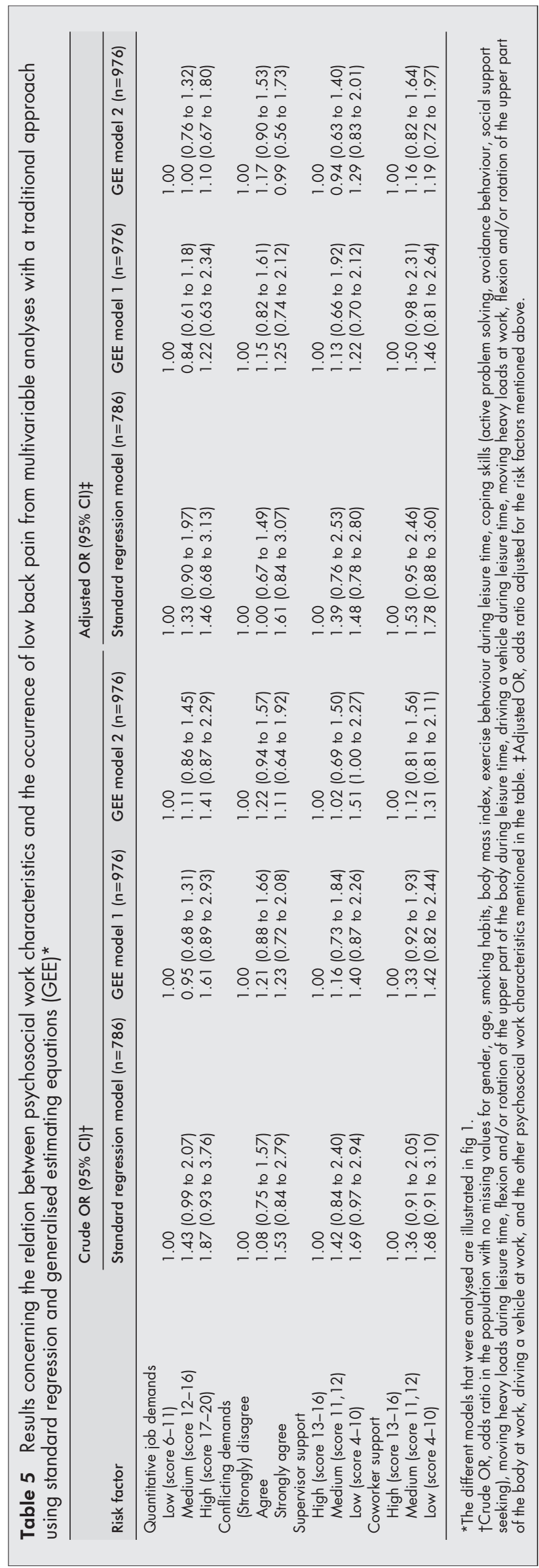

ratios were obtained in the analysis of GEE model 2, which was considered a priori to be the most appropriate, than in the analysis of the standard regression model.

The difference in results of GEE models 1 and 2 suggests that changes in exposure have apparently occurred in the case of flexion and/or rotation of the upper part of the body. The overview of changes in reported exposure between subsequent measurements also showed that the exposure to flexion and/or rotation of the upper part of the body changed one or more levels for $30-40 \%$ of workers between subsequent measurements. An additional analysis of the data on exposure showed that only $49 \%$ of workers reported the same level of exposure for flexion and/or rotation of the upper part of the body, both at baseline and at the first two follow up measurements (data not shown).

For moving heavy loads, taking into account the time varying nature of the exposure did not change the findings. The adjusted odds ratios of GEE model 2 and GEE model 1 were quite similar. This can be explained by the relative stability of the variable of moving heavy loads over the follow up period. The overview of changes in reported exposure between subsequent measurements showed that the exposure to moving heavy loads changed one or more levels for only $10-15 \%$ of workers between subsequent measurements. For this variable, $76 \%$ of workers reported the same level of exposure, both at baseline and at the first two follow up measurements (data not shown).

In comparison with the results for the work related physical factors, the results for the psychosocial work characteristics were rather heterogeneous. In addition, no clear evidence of a relation between any of the psychosocial work characteristics studied and reported low back pain was found with either the traditional approach or the GEE method. Therefore, the possibility of making inferences based on the observed differences between the models was limited. In general, in the analysis of GEE model 2, the model that was considered a priori to be the most appropriate, weaker associations for the psychosocial work characteristics were found than in the analysis of the traditional standard regression model.

For both high quantitative job demands and high conflicting demands, the comparison of GEE model 2 with GEE model 1 showed that taking into account the time varying nature of the exposure led to a decrease of the odds ratios for these psychosocial work characteristics towards the neutral value. With regard to the social support variables, comparison of GEE model 2 with GEE model 1 showed that taking into account the time varying nature of the exposure led to an increase in the odds ratio for low supervisor support, whereas the odds ratio for low coworker support decreased towards the neutral value. Apparently, the influence of taking into account the time varying nature of the exposure is not the same for all psychosocial work characteristics. This cannot be explained by differences in the stability of these variables over the follow up period. For all psychosocial work characteristics, no more than $60-70 \%$ of workers reported the same level of exposure at subsequent measurements, and only approximately half of the workers reported the same level of exposure, both at baseline and at the first two follow up measurements (data not shown).

In addition to differences in the magnitude of the odds ratios, differences in the precision of the odds ratios for both physical and psychosocial factors were also observed. The confidence intervals of the odds ratios resulting from the GEE analyses were smaller than those of the odds ratios resulting from the traditional standard regression analyses. This can be explained by the fact that all available outcome data can be included in the GEE method. In the present study, 786 workers with data on the occurrence of low back pain for all three annual follow up measurements were included in the traditional standard regression analyses, while 976 workers with data on the occurrence of low back pain for at least one 
of the follow up measurements were included in the GEE analyses. For the majority of these workers, more than one observation was also included in the analyses, which resulted in even more power. Only for moving heavy loads did the increase in power result in a different conclusion with regard to the presence of a statistically significant relationship with low back pain.

\section{Limitations and potential sources of bias}

Only the two GEE models included in the present analysis were taken into consideration, although other possibilities do exist. The time lag model, the preferred model for the present data, takes into account the temporal sequence of cause and effect, and this model can also be extended to models with different time lags. By repeatedly measuring exposure and outcome, the biologically relevant exposure window at some fixed interval of time relative to the outcome event can be defined. ${ }^{8}$ The length of the exposure window and its temporal position relative to the outcome event will depend on the supposed causal mechanism for the exposure of interest. When the understanding of disease aetiology is too limited to specify a credible temporal relationship, different time lags can be studied to obtain more insight into the hypothesised relationships. ${ }^{18}$ It is beyond the scope of this paper to examine all these possibilities, but it is clear that modelling approaches such as the GEE method, make it feasible to explore these issues in the context of epidemiological cohort studies with repeated measurements of exposure and health data. ${ }^{19}$

One can imagine that, especially with regard to the psychosocial work characteristics, the postulation of different time lags could provide insight into the nature of the relationship of these variables with low back pain. The inclusion of an appropriate time lag is especially important for exposures that are relatively unstable. The psychosocial work environment is clearly subject to change. It can be hypothesised that changes in the psychosocial work environment occur even more frequently than changes in the physical work environment, and that psychosocial work characteristics may have a more short term effect on low back pain. Consequently, shorter intervals between the repeated measurements would be necessary to make an adequate assessment of the role of psychosocial work characteristics. Another explanation for the difference in results for physical and psychosocial exposures might be that the physical exposures have a much more immediate effect, whereas the psychosocial exposures need to accumulate over time before they start to have an effect on low back pain.

In the GEE analyses, a maximum of 1116 workers with data on the occurrence of low back pain for at least one of the follow up measurements could be included. This was done under the assumption that the pattern of missing data on the occurrence of low back pain during the follow up is random. It is not possible to verify completely whether this assumption holds. However, the incidence of low back pain at the first follow up did not differ for those workers who were lost to follow up after this specific measurement (data not shown).

The GEE analyses reported in the present paper were performed using an exchangeable working correlation matrix for reasons given in the methods section. In general, the choice for the correlation structure has to be based on the correlation of the repeated outcome measures in the dataset. However, in case of a binary outcome, the value of a correlation coefficient as a measure of association is limited. Fortunately, the GEE analysis is known to be quite robust for the choice of a "wrong" correlation structure. ${ }^{67}$ The analyses presented in this paper were also performed using an unstructured working correlation which assumes unconstrained pairwise correlations. Use of this structure gave virtually identical estimates of the odds ratios and accompanying confidence intervals compared to those found using the exchangeable structure.
As mentioned in the introduction, the GEE method is one of the methods that can be used for analysis of repeated measurements data. Other potential methods for dealing with these kinds of data exist, such as multilevel models or transitional models. An important reason to choose for a comparison of a traditional approach using standard regression with GEE in this paper, was that GEE is becoming increasingly popular for the analysis of longitudinal data and that the possibility of performing GEE is included in regular available software. This choice, however, does not imply that the authors consider GEE the only and best available option for the kind of data at hand in this paper. One of the problems of using multilevel models is that these models have not been completely worked out for dichotomous outcome variables. ${ }^{20}$ This results in differences in findings for the same dataset, depending on the specific software package used.

The data sets used for the analysis of GEE models 1 and 2 can also be analysed using standard logistic regression. ${ }^{79}$ Comparison of the results of such an analysis with the results of GEE gives insight into the effect of taking into account the within subject correlation. For all risk factors under study in this paper a standard regression analysis on the data of GEE models 1 and 2 gave only slightly different odds ratios than GEE. The estimated confidence intervals were in general smaller for GEE model 1 and similar or slightly greater for GEE model 2.

A potential bias that is specifically related to longitudinal studies in which both exposure variables and outcome variables are allowed to vary over time, is feedback bias, which implies that earlier outcomes may affect subsequent exposures. ${ }^{8}$ As explained by Eisen, the GEE method cannot control for this bias.

It could be argued that the number of workers used in the standard regression model, and consequently the power, could have been made larger by including the 52 workers that had missing data on the outcome, but reported low back pain at one of the follow up measurements in which they participated. This was decided against as it might introduce bias, because it would be more likely to include a person with missing data if they did have back pain.

A specific limitation of the analyses of work related physical risk factors described in the present paper is that all measurements were based on self reports. In the SMASH study, physical load at work was also quantified by means of analyses of video recordings. However, as these measurements were not repeated at the annual follow up measurements, these data are not included in this paper. In general, the results of the present analyses, based on self reported measures of physical load at work, are in accordance with the results of the previously reported analyses of quantified measures based on the observations of baseline video recordings. ${ }^{10}$

\section{Conclusion}

In conclusion, the results of this study clearly show that there are differences between the two analytical approaches applied, in both magnitude and precision of the calculated odds ratios. Compared with the traditional approach using standard regression analysis, GEE analysis with a time lag model, in which the time varying nature of both the exposure and the outcome is taken into account, revealed stronger associations for the work related physical risk factors studied and weaker associations for the psychosocial work characteristics. Moreover, for moving heavy loads, conclusions on the presence or absence of a statistically significant relationship with low back pain differed, depending on the analytical approach used. The analyses of another model with the GEE method provided insight into the specific effect of taking into account the time varying nature of the exposure. This appeared to have an influence on the results.

This paper clearly shows that in the design and analysis of prospective cohort studies on work related risk factors for 
musculoskeletal symptoms, the incorporation of repeated measurements of exposure should be considered. GEE analysis provides a means of dealing with this kind of data. An additional advantage is that changes in outcome, that apparently occur in case of an episodic outcome event, can also be taken into account. To encourage researchers to carry out such studies, discussions should be initiated to determine the appropriate intervals between the repeated measurements and also the appropriate number of repeated measurements needed to study both work related physical and, in particular, psychosocial risk factors for low back pain.

\section{ACKNOWLEDGEMENTS}

The Study on Musculoskeletal disorders, Absenteeism, Stress, and Health (SMASH) was financially supported by the Dutch Ministry of Social Affairs and Employment, the Dutch Ministry of Public Health, Welfare and Sports, and the Dutch National Institute for Social Security.

\section{APPENDIX: REGRESSION EQUATIONS FOR THE MODELS ILLUSTRATED IN FIG 1 Standard regression model:}

$\operatorname{Ln}\left(\frac{\mathrm{P}\left(\mathrm{Y}_{\mathrm{i}}=1\right)}{\mathrm{P}\left(\mathrm{Y}_{\mathrm{i}}=0\right)}\right)=\beta_{0}+\beta_{1} \mathrm{x}_{1 \mathrm{i}, \mathrm{to}}+\ldots+\beta_{\mathrm{p}} \mathrm{x}_{\mathrm{pi}, \mathrm{to}}$

where: $Y_{i}=$ binary outcome for subject $\mathrm{i}, \beta_{0}=$ intercept, $\mathrm{x}_{\mathrm{it}, \mathrm{t}}=$ independent variable $x_{1}$ of subject $i$ at time $t=0, \exp \left(\beta_{1}\right)=$ odds ratio of independent variable $\mathrm{x}_{1}, \mathrm{x}_{\mathrm{p}, 10}=$ independent variable $\mathrm{x}_{\mathrm{p}}$ of subject $\mathrm{i}$ at time $t=0, \exp \left(\beta_{p}\right)=$ odds ratio of independent variable $x_{p}$.

\section{GEE model 1:}

$\operatorname{Ln}\left(\frac{\mathrm{P}\left(\mathrm{Y}_{\mathrm{it}}=1\right)}{\mathrm{P}\left(\mathrm{Y}_{\mathrm{it}}=0\right)}\right)=\beta_{0}+\beta_{1} \mathrm{x}_{\mathrm{li}, \mathrm{to}}+\ldots+\beta_{\mathrm{p}} \mathrm{x}_{\mathrm{pi}, \mathrm{t} 0}$

where: $Y_{i t}=$ binary outcome for subject $\mathrm{i}$ at time $\mathrm{t}, \beta_{0}=$ intercept, $\mathrm{x}_{\mathrm{it}, \mathrm{t}}$ $=$ independent variable $x_{1}$ of subject $\mathrm{i}$ at time $\mathrm{t}=0$, $\exp \left(\beta_{1}\right)=$ odds ratio of independent variable $x_{1}, x_{p i t 0}=$ independent variable $x_{p}$ of subject $i$ at time $t=0, \exp \left(\beta_{p}\right)=$ odds ratio of independent variable $x_{p}$.

\section{GEE model 2:}

$\operatorname{Ln}\left(\frac{\mathrm{P}\left(\mathrm{Y}_{\mathrm{it}}=1\right)}{\mathrm{P}\left(\mathrm{Y}_{\mathrm{it}}=0\right)}\right)=\beta_{0}+\beta_{1} \mathrm{x}_{1 \mathrm{i}, \mathrm{t}-\mathrm{l}}+\ldots+\beta_{\mathrm{p}} \mathrm{x}_{\mathrm{pi}, \mathrm{t}-\mathrm{l}}$

where: $Y_{\mathrm{it}}=$ binary outcome for subject $\mathrm{i}$ at time $\mathrm{t}, \beta_{0}=$ intercept, $\mathrm{x}_{\mathrm{i}, \mathrm{t}-\mathrm{1}}$ $=$ independent variable $x_{1}$ of subject $i$ at time $t-1, \exp \left(\beta_{1}\right)=$ odds ratio of independent variable $\mathrm{x}_{1}, \mathrm{x}_{\mathrm{p}, \mathrm{t}-\mathrm{1}}=$ independent variable $\mathrm{x}_{\mathrm{p}}$ of subject $\mathrm{i}$ at time $t-1, \exp \left(\beta_{\mathrm{p}}\right)=$ odds ratio of independent variable $\mathrm{x}_{\mathrm{p}}$.

\section{Authors' affiliations}

W E Hoogendoorn, P M Bongers, TNO Work and Employment, Hoofddorp, Netherlands

H C W de Vet, J W R Twisk, W van Mechelen, L M Bouter, Institute for Research in Extramural Medicine, Vrije Universiteit Medical Centre, Amsterdam, Netherlands

\section{REFERENCES}

1 Bongers PM, Winter CR de, Kompier MA, et al. Psychosocial factors at work and musculoskeletal disease [review]. Scand J Work Environ Health 1993;19:297-312.

2 Burdorf A, Sorock G. Positive and negative evidence of risk factors for back disorders [review]. Scand J Work Environ Health 1997:23:243-56.

3 Bernard BP, ed. Musculoskeletal disorders and workplace factors. A critical review of epidemiologic evidence for work-related musculoskeletal disorders of the neck, upper extremity, and low back. Cincinnati, $\mathrm{OH}$ : National Institute for Occupational Safety and Health, US Department of Health and Human Services, 1997.

4 Riihimäki H. Musculoskeletal diseases-a continuing challenge for epidemiologic research. Scand J Work Environ Health 1999;25(suppl 4):31-5.

5 Hoogendoorn WE, Poppel MNM van, Bongers PM, et al. Physical load during work and leisure time as risk factors for back pain [review]. Scand J Work Environ Health 1999;25:387-403.

6 Liang K-Y, Zeger SL. Longitudinal data analysis using generalized linear models. Biometrika 1986;73:13-22.

7 Diggle PJ, Liang K-Y, Zeger SL. Analysis of longitudinal data. New York: Oxford University Press, 1994

8 Eisen EA. Methodology for analyzing episodic events. Scand J Work Environ Health 1999;25(suppl 4):36-42.

9 Twisk JWR. Different statistical models to analyze epidemiological observational longitudinal data: an example from the Amsterdam Growth and Health Study. Int J Sports Med 1997;18:S216-24.

10 Hoogendoorn WE, Bongers PM, Vet HCW de, et al. Flexion and rotation of the trunk and lifting at work are risk factors for low back pain: results of a prospective cohort study. Spine 2000;25:3087-92.

11 Hoogendoorn WE, Bongers PM, Vet HCW de, et al. Psychosocial work characteristics and psychological strain in relation to low-back pain. Scand J Work Environ Health 2001;27:258-67.

12 Godin G, Jobin J, Bouillon J. Assessment of leisure time exercise behavior by self-report: a concurrent validity study. Can J Publ Health 1986:77:359-62.

13 Schreurs PJG, Willige G van der, Tellegen B, et al. The Utrecht Coping List: UCL Manual (in Dutch). Lisse: Swets \& Zeitlinger, 1988

14 Karasek R. Job Content Instrument users guide: revision 1.1. Los Angeles: Department of Industrial and Systems Engineering, University of Southern California, 1985

15 Hildebrandt VH, Douwes M. Physical load and work: questionnaire on musculoskeletal load and health complaints (in Dutch). Voorburg: Ministry of Social Affairs and Employment, 1991:S122-3.

16 Kuorinka I, Jonsson B, Kilbom A, et al. Standardised Nordic questionnaire for the analysis of musculoskeletal symptoms. Applied Ergonomics 1987; 18:233-7.

17 SAS Institute Inc. SAS/STAT Software: Changes and enhancements through release 6. 12. Cary, NC: SAS Institue Inc., 1997.

18 Willett WC, Colditz GA. Approaches for conducting large cohort studies. Epidemiol Rev 1998;20:91-9.

19 Tager IB. Outcomes in cohort studies. Epidemiol Rev 1998;20:15-28.

20 Goldstein H. Multilevel statistical models, 2 nd edn. London: Edward Arnold, 1995. 